Rapport - Société canadienne d'histoire de l'Église catholique

\title{
Nomination et sacre de Mgr de Laval
}

\section{Georges-Edouard Demers}

Volume 25, 1957-1958

URI : https://id.erudit.org/iderudit/1007440ar

DOI : https://doi.org/10.7202/1007440ar

Aller au sommaire du numéro

Éditeur(s)

La Société canadienne d'histoire de l'Église catholique

ISSN

0318-6148 (imprimé)

1927-7075 (numérique)

Découvrir la revue

Citer cet article

Demers, G.-E. (1957). Nomination et sacre de Mgr de Laval. Rapport - Société canadienne d'histoire de l'Église catholique, 25, 13-32.

https://doi.org/10.7202/1007440ar

Tous droits réservés @ La Société canadienne d'histoire de l'Église catholique, 1958
Ce document est protégé par la loi sur le droit d'auteur. L’utilisation des services d'Érudit (y compris la reproduction) est assujettie à sa politique d'utilisation que vous pouvez consulter en ligne.

https://apropos.erudit.org/fr/usagers/politique-dutilisation/ 


\section{Nomination et sacre de Mgr de Laval}

Dès ses tendres années, le jeune François de Laval avait vu se lever devant lui la vision du sacerdoce. Le climat spirituel intensément imprégné d'inspiration missionnaire dans lequel se développèrent les étapes de sa formation, devait donner à ce rêve, d'abord confus, la forme précise de l'apostolat en pays de mission.

En 1631, quand il arriva à La Flèche, à peine âgé de huit ans, pour faire ses humanités, le collège royal Henri IV vibrait encore des émotions apostoliques ressenties aux fêtes solennelles qui avaient marqué la canonisation de saint Ignace et de saint François Xavier (1622). Dès lors une forte orientation des esprits vers l'idéal missionnaire offrait aux âmes généreuses un aliment à leurs aspirations. La gloire de Dieu, dont s'imprègne si profondément la spiritualité ignatienne, ne semblait plus avoir pour bien des jeunes enthousiasmes qu'une réalisation possible: les missions. Et, de toutes les missions, aucune n'avait le charme fascinateur de celles du Canada.

Le Père Ministre du collège contribuait largement à entretenir cette ardeur pour la cause religieuse canadienne. Expulsé depuis peu de temps de la Nouvelle-France par la conquête anglaise, le P. Ennemond Massé avait transporté à La Flèche le zèle apostolique dont il était dévoré. Son influence sur la jeunesse étudiante de Henri IV fut prépondérante et donna naissance à un généreux essaim d'ouvriers évangéliques, qui devaient un jour remplir de leurs exemples les annales religieuses de la Nouvelle-France.

Plusieurs futurs missionnaires du Canada se trouvaient au collège de La Flèche en même temps que François de Laval. Claude d'Ablon, Jacques Buteux, Simon Lemoyne, Charles Turpin, Jean Dolbeau, Jacques Bonin, tous scolastiques de la Compagnie de Jésus, y faisaient leur philosophie ou leur théologie. Le P. Claude Pijart et le P. Gabriel Lallement étaient surveillants des écoliers ${ }^{1}$.

C'est sous cette influence que le jeune François de Laval sentit pour la première fois de "l'inclination" à consacrer sa vie aux missions canadiennes ${ }^{2}$.

En automne de 1641, sa philosophie terminée, il partait pour Paris afin d'y faire sa théologie chez les Jésuites du collège de Clermont.

A Clermont, le même souffle sacré produisait en faveur des missions canadiennes le même engouement irrésistible, lequel d'ailleurs avait

1 Camille de Rochemonteix, s.j., Les Jésuites et la Nouvelle-France au XVII siècle, Paris, 1895-1896, vol. II, p. 240.

2 Mémoire touchant feu M. de Laval, 1708, Arch. du Séminaire de St-Sulpice de Paris, Documents pour servir à l'histoire de l'Eglise du Canada, vol. I, liasse de papiers sur $\mathrm{M}^{\mathrm{gr}}$ de Laval, fol. 3 . 
gagné tout Paris, le Paris courtisan, le Paris populaire, tout comme le Paris religieux. Les Relations des Jésuites, qui depuis 1632 venaient chaque année répéter devant le public français leur refrain de confiance en la petite colonie d'outre-mer, nourrissaient cette ardeur. L'émotion fut à son comble quand, au cours de la troisième année de théologie de François de Laval (1643-1644), le P. Isaac Jogues arriva en France, présentant ses pauvres mains horriblement mutilées par les Iroquois. Les maisons jésuites de Paris reçurent le martyr comme un témoin vivant du rude apostolat en terre canadienne, et les consciences généreuses furent vivement remuées de respectueuse admiration.

L'âme de Laval vibrait à l'unisson de cette ardeur apostolique, quand un incident tragique vint subitement traverser ses projets et mettre même en péril sa vocation au sacerdoce. Par la mort de son père, en 1636, et par celles de ses deux frères aînés, en 1644 et 1645 , il devenait subitement chef de sa famille, seigneur de Montigny, Montbaudry, Alaincourt et Révercourt, et chargé de l'administration temporelle de ses seigneuries. Si le jeune clerc dût rentrer à Montigny-sur-Avre, le lieu de sa naissance, il n'est pas juste d'affirmer avec son premier biographe, Bertrand de Latour, qu'il a quitté alors l'état ecclésiastique ${ }^{3}$; car, déjà nommé chanoine $d^{\prime}$ Evreux par son oncle, $M^{\mathrm{gr}}$ de Péricard, en 1637, transféré à un autre bénéfice canonial en 1639, il garda ce dernier sans interruption jusqu'en $1648^{4}$, alors qu'il était prêtre depuis un an.

Entre temps, l'abbé de Montigny (c'est sous ce nom qu'on l'appellera désormais) rentrait à Paris, recevait l'onction sacerdotale le $1^{\text {er }}$ mai $1647^{5}$ et devenait grand archidiacre d'Evreux le 7 décembre $1649^{6}$. L'année suivante, il prenait en Sorbonne sa licence en droit canonique ?.

C'est au cours du séjour de François de Laval à Paris que se forma cette mystérieuse association connue sous la non moins mystérieuse dénomination de $A a$, et qui eut, tant sur la vie spirituelle du futur évêque de Québec et sur l'orientation de ses destinées, que sur la formation du premier clergé canadien, une telle influence, qu'il importe d'en signaler ici au moins les notes essentielles.

3 Bertrand de Latour, Mémoires sur la vie de M. de Laval, Cologne, 1761, p. 1. Un' ms. de ce premier volume de Latour est conservé à la Bibliothèque municipale d'Amiens, ms. 468, $2^{\mathrm{e}}$ partie.

4 Pouillé d'Evreux, Arch. départementales de l'Eure (Evreux), G 22, AG 35, ff. 247, 506-507; Auguste Gosselin, Le Vénérable François de Montmorency-Laval, Québec, 1923, p. 34.

5 Nous n'avons pu retracer l'acte d'ordination sacerdotale de $\mathrm{M}^{\mathrm{gr}} \mathrm{de}$ Laval. Cependant plusieurs documents contemporains nous indiquent l'année 1647 comme celle de son sacerdoce. Signalons, entre autres, une lettre du P. Jérôme Gravier, datée de 1697, à $\mathrm{M}^{\mathrm{gr}}$ de Laval, dans laquelle il fait allusion au cinquantenaire d'ordination du prélat qu'on avait célébré cette année-là. (Original aux Arch. du Séminaire de Québec, Lettres N, $\mathbf{n}^{\circ} 131$ ). Quant à la date du $1^{\text {er }}$ mai, nous croyons pouvoir l'adopter à la suite de l'abbé Auguste Gosselin, Henri de Bernières, premier curé de Québec, Evreux, 1896 p. 150, note 1.

- Pouillé d'Evreux, Arch. départementales de l'Eure (Evreux), G 22, AG 35, fol. 145.

7 c...in jure canonico licentiatus ab anno millesimo sexcentesimo quadragesimo nono.» Informations canoniques de $\mathbf{M}^{\mathrm{gr}}$ ide Laval, 1657. Copie aux Arch. du Séminaire de Québec, Evêques de Québec, $n^{\circ} 183$. 
L'Aa, qu'il faut distinguer de la Compagnie du Saint-Sacrement ${ }^{8}$, est une association secrète, formée au sein des grandes Congrégations mariales, dont les membres avaient résolu de mener une vie chrétienne très fervente par la stricte observance des règles de la Congrégation, par des exercices de piété et de pénitence faits en commun et par la pratique des ouvres de charité sous la protection de la Sainte Vierge. Le secret qui unit les membres entre eux, n'a d'autre but que de rendre plus facile la réalisation de cet idéal. Les premiers essais de cette "Congrégation * fermée furent faits au collège de La Flèche en 1630 ou 1632, mais l'Aa principale fut celle de Paris, fondée en 1643 et communément appelée Société des Bons Amis; toutes les autres fondées dans la suite en France, en Bavière, en Italie et au' Canada, où tous les prêtres du Séminaire de Québec étaient membres de cette association, ne furent que des filiales de celle de Paris. Au tout début, les Bons Amis se contentèrent de se réunir chaque semaine; mais bientôt ils décidèrent de vivre en commun dans un appartement loué à cet effet ${ }^{9}$.

François de Laval, déjà consacré à la Sainte Vierge dans la Congrégation mariale du collège de La Flèche ${ }^{10}$, fut un des premiers membres de l'Aa. Si on en croit le mémoire pamphlétaire préparé en 1677 par Frontenac, il en aurait été supérieur ou commis pendant un certain temps ${ }^{11}$. En tout cas, c'est à la Société des Bons Amis qu'il a developpé sa toute particulière dévotion à la Sainte Vierge, à la Sainte Famille et aux saints Anges, patrons de l' $\boldsymbol{A} a$, son grand esprit de pauvreté et ce singulier attachement au Saint-Siège, qui reste une des caractéristiques particulières de sa personnalité spirituelle ${ }^{12}$.

C'est aussi par l'entremise de l'Aa que la Providence lui signifia sa vocation d'évêque missionnaire.

Et voici dans quelles circonstances.

8 Ferdinand Cavallera, s.j., Le ms. 277 de la Bibliothèque publique de la ville de Toulouse et l'Aa toulousaine, dans Revue d'ascétique et mystique, vol. XVI (1935), pp. 299-303.

9 Sur l'Aa, cf. Latour, op. cit., pp. 2-5. 32-33; Comte de Bégouen, Une société secrète émule de la Compagnie du Saint-Sacrement, l'Aa de Toulouse, ParisToulouse, 1913; Ferdinand Cavallera, Aux origines de la Société des missions étrangères. L'Aa de Paris, dans Bulletin de littérature ecclésiastique, Toulouse, vool. XXXIV (1933), pp. 173-186, 206-226; vol. XXXV (1934), pp. 17-31, 71-96; M. Villar, art. Aa dans Dictionnaire de spiritualité, vol. I, Paris, 1937, col. 1; Robert Rouquette, art. Congrégations secrètes, op. cit., vol. II, Paris, 1953, col. 1491-1507.

10 Nous ne savons pas exactement à quelle date François de Laval fut admis dans la congrégation mariale de La Flèche. Selon le $P$. de Rochemonteix (op. cit., vol. II, p. 241), ce ne put être avant son année de troisième (16351636), aucun élève ne pouvant être reçu congréganiste avant d'avoir été promu à cette classe. Le 2 février $1673, M^{\mathbf{g r}^{\mathbf{r}}}$ de Laval renouvelait sa consécration à Paris, comme en fait foi un parchemin signé de sa main et inséré dans un recueil de formules de consécration, conservé aux Archives Nationales de Paris, MM, vol. 649, fol. 20.

11 Mémoire de Frontenac, Bibl. Nat. de Paris, Claraimbault, vol. 1016, fol. 45.

12 Une enquête menée en 1664 par des juristes gallicans signale la Congrégation du P. Bagot, donc l'Aa, parmi les associations plus particulièrement attachées au Saint-Siège (Charles Gérin, Recherches historiques sur l'Assemblée du clergé de France de 1682, Paris, 1869, p. 488). 
Au début de l'année 1653, un célèbre Jésuite, missionnaire en Extrême-Orient, le P. Alexandre de Rhodes, arrivait à Paris. Sur l'instance de ses confrères de mission, il avait entrepris le voyage d'Europe pour recruter de nouveaux ouvriers apostoliques et obtenir du Souverain Pontife l'envoi d'évêques missionnaires dans ses lointaines missions. Il fallait là-bas créer un clergé indigène et assurer l'administration du sacrement de confirmation à des milliers de néo-chrétiens qui en étaient privés. Mandaté par le Pape Innocent $\mathrm{X}$ et encouragé par le Général de la Compagnie de Jésus, il avait d'abord cherché en Italie des candidats dignes d'être présentés à l'épiscopat missionnaire; mais ses efforts avaient été vains ${ }^{13}$. Il venait maintenant en France dans l'espoir de trouver les âmes généreuses qui accepteraient de s'expatrier pour l'extension du Royaume de Dieu.

S'étant ouvert de son projet au P. Jean Bagot, jésuite comme lui, celui-ci lui prépara une rencontre avec les jeunes congréganistes de l'Aa. L'entrevue eut lieu à l'occasion d'un dîner intime dans le petit logis où ils habitaient, rue Coupeaux.

Le P. de Rhodes leur parla avec enthousiasme de ses projets. La semence tombait en bonne terre; si bien que tous, spontanément, s'offraient à partir pour l'Asie, promettant même " de toutes leurs forces à s'y employer toute leur vie à leurs propres frais ${ }^{14}$ ".

A l'issue de ce premier entretien, le P. de Rhodes ne craignait pas d'affirmer: "J'ai trouvé dans ces jeunes gens des dispositions plus parfaites que celles que j'ai recherchées dans les séminaires et autres lieux d'Europe ${ }^{15}$.»

Trois candidats furent alors choisis pour les futurs vicariats apostoliques du Tonkin, de la Chine et de la Cochinchine: François Pallu, chanoine de Tours, Pierre Piques, docteur en Sorbonne, et François de Laval, grand archidiacre d'Evreux, qui devait être proposé pour le Tonkin.

Les noms des trois aspirants furent présentés au Conseil de Conscience de la Reine régente, à qui était dévolu le privilège exclusif de demander à Rome la nomination de nouveaux évêques.

On sait que depuis les débuts du Conseil de Conscience, fondé par Anne d'Autriche pour le temps de la régence, saint Vincent de Paul y exerçait un rôle de première importance. S'il n'en avait pas lui-même la présidence, il n'en était pas moins la cheville ouvrière; c'est lui qui prenait les informations des affaires difficiles, étudiait les dossiers, présentait les rapports ${ }^{16}$. L'intègre Monsieur Vincent, toujours si zélé pour tout ce qui regardait la formation du clergé, se montrait particulièrement circonspect lorsqu'il fallait proposer au Souverain Pontife la nomination de nouveaux évêques; dès lors, les éloges des candidats à l'épiscopat prennent, dans les requêtes signées par lui, une valeur que

Arch. Nat. de Paris, MM 529, fol. 1.

Ibid., fol. 3.

Mathieu, Vie de Henri-Marie Boudon, Besançon, 1837, p. 54.

16 Coste, Monsieur Vincent, Paris, 1932, vol. II, p. 106. 
l'on ne saurait sous-estimer. Aussi ne croyons-nous pas inutile de citer ici les deux suppliques présentées alors par le Conseil de Conscience; l'éloge qu'on y fait des trois candidats proposés s'adresse en particulier à François de Laval.

Dans une première supplique au Pape Innocent $\mathrm{X}$ datée de juillet 1653, Monsieur Vincent et les autres signataires affirmaient: « Nous avons à Paris plusieurs prêtres séculiers qui seraient capables de remplir ces fonctions. Ils sont recommandables par la pureté de leurs mœurs, leur zèle, leur prudence et leur doctrine; ils sont prêts du reste à subir l'examen de personnes que Votre Sainteté voudrait bien désigner ${ }^{17}$."

Plus explicite encore est la seconde requête envoyée au Saint-Siège quelques mois plus tard par le Conseil de Conscience et signée encore par Monsieur Vincent. Après avoir exposé que le nombre insuffisant de missionnaires dans les pays de la Chine et de la Cochinchine postule la nomination d'évêques missionnaires, le document ajoute: "Nous avons ici sous la main trois prêtres choisis, d'une probité connue, tout dévoués à cette cause si dure et prêts à entreprendre pour le Christ ce voyage plein de dangers et à travailler toute leur vie sans répit dans ces royaumes lointains ${ }^{18}$."

Entre temps le Nonce de Paris, Nicolas Bagny, reçut l'ordre du Saint-Siège de prendre sur les trois évêques proposés les informations canoniques exigées par le concile de Trente ${ }^{19}$.

Six témoins furent entendus, le 5 novembre 1653, qui attestèrent que François de Laval possédait toutes les qualités de doctrine, de piété, de zèle et de prudence, pour que l'Eglise et le bien des âmes retirent un profit notable de sa promotion à l'épiscopat en pays de mission ${ }^{20}$.

Pour sa part, l'abbé de Montigny resta étranger à toutes les démarches qui l'acheminaient à l'honneur de l'épiscopat. Dans le silence, il se préparait à sa future mission apostolique par le détachement des biens terrestres et par un renouvellement de vie intérieure.

C'est ainsi qu'en décembre 1653 il se démit, en faveur de son ami Henri Boudon, de son archidiaconé d'Evreux ${ }^{21}$; l'année suivante, il fit une résignation complète de tous ses biens de famille et de ses seigneuries, en cédant à son frère cadet Jean-Louis ses titres et ses droits de naissance ${ }^{22}$. Puis, libre de toute attache, il alla se réfugier dans la pieuse solitude de l'Ermitage de Caen, où, sous la direction du mystique M. de

17 Arch. du Séminaire des missions étrangères de Paris, vol. 114, p. 434.

18 Coste, Saint Vincent de Paul, correspondance, entretiens, documents, Paris, 1920-1925, vol. V, p. 11.

19 Sur les négociations entre la cour de Paris et le Saint-Siège pour la nomination des trois vicaires apostoliques, cf. Baudiment, François Pallu, Paris, 1934, pp. 30-39.

20 Arch. de la Propagande, Scritture riferite nelle cong. gen., vol. 317, ff. 126-137.

21 Pouillé d'Evreux, Arch. départ. de l'Eure (Evreux), G 22, AG 35, fol. 145.

22 Informations canoniques, 1653, Arch. de la Propagande, Scritture riferite nelle cong. gen., vol. 137, ff. 126-137; Latour, op. cit., p. 2. 
Bernières-Louvigny, il s'appliqua à retremper les forces de son âme dans la prière, la mortification et les œuvres de la charité chrétienne.

L'Ermitage de Caen. Nous croyons nécessaire de faire une brève incursion dans cette maison de retraite, dont l'atmosphère de contemplation et de pénitence marqua profondément de son empreinte la spiritualité de $\mathrm{M}^{\mathrm{gr}}$ de Laval ${ }^{23}$; empreinte si forte que Marie de l'Incarnation n'hésitera pas à écrire, quand en 1659 elle aura rencontré le prélat: "Il est ami intime de $M$. de Bernières avec qui il a demeuré quatre ans par dévotion; aussi, ajoute-t-elle, ne se faut-il pas trop étonner, si ayant fréquenté cette école, il est parvenu au sublime degré d'oraison où nous le voyons ${ }^{24}$."

Jean de Bernières-Louvigny était un de ces chrétiens fervents, nombreux au $\mathrm{XVII}^{\circ}$ siècle, qui, soit par crainte des responsabilités, soit par humilité ou par un secret dessein de Dieu, avaient renoncé au sacerdoce ou à la vie religieuse pour pratiquer dans le monde les plus haute vertus. Beaucoup de laïques et de prêtres se mettaient sous la direction de ces pieuses personnes, à qui ils confiaient le soin de leur âme. "Chose curieuse, remarque l'abbé Henri Bremont, le $\mathrm{XVII}^{\circ}$ siècle qui, grâce à l'école française, a élevé si haut l'idée du sacerdoce chrétien, trouve naturel qu'une Madame Alarie, qu'un M. de Renty, qu'un M. de Bernières, se chargent de la direction des âmes. L'Ordre le plus précautionné, la Compagnie de Jésus, ne trouve à cela aucun inconvénient ${ }^{25}$.»

Pour recevoir ses disciples, Jean de Bernières avait fait construire de 1646 à 1649 une maison de retraite à Caen, où les ermites résidents ou des amis de passage trouvaient, avec les sages directives de leur maître, le silence nécessaire à la prière et à la méditation ${ }^{26}$.

François de Laval demeura quatre ans à l'Ermitage de Caen, vraisemblablement de 1654 à 1658 . En vue de se disposer à son ministère futur, il se soumit à la dure ascèse des ermites: prières prolongées, veilles, macérations corporelles, soin des malades dans les hôpitaux, pèlerinages sous le déguisement d'un pauvre ${ }^{27}$.

23 Georges-E. Demers, Un guide spirituel de $M^{g r}$ de Laval, Jean de BernièresLouvigny, dans Nouvelle Abeille, vol. I (1931-1935), pp. 279-281.

24 Marie de l'Incarnation à son fils, dom Claude Martin, 1659, Lettres de la Vénérable Mère Marie de L'Incarnation, Paris, 1681, p. 541.

25 Henri Bremond, Histoire littéraire du sentiment religieux en France..., vol. VI, Paris, 1922, p. 230.

26 On a émis quelques doutes sur l'orthodoxie doctrinale de l'Ermitage, la traduction italienne faite en $1666 \mathrm{du}$ Chrétien intérieur (Un solitaire, Le Chrestien intérieur..., Rouen, 1660), livre attribué à M. de Bernières, ayant été condamné par décret de l'Index le 26 juin 1689. Mais des études récentes ont démontré que le livre, composé par de mauvais disciples après la mort du mystique de Caen, n'expose pas la vraie doctrine du maître, laquelle s'avère, à une étude minutieuse, exempte de toute trace d'erreur théologique (A. Saudreau, La vie d'union à Dieu, Angers, 1921, p. 354; Maurice Sauriau, Le mysticisme en Normandie au XVII ${ }^{\circ}$ siècle, Paris, 1923; Raoul Heurtevent, L'œuvre spirituelle de Jean de Bernières, Paris, 1938).

27 Latour, op. cit., pp. 7-8. C'est probablement à l'occasion d'un de ces pieux pèlerinages qu'il visita Marie des Vallées, la *sainte âme de Coutances, comme on l'appelait, pour laquelle, selon Latour, (op. cit., pp. 30-31), il 
En réalité, François de Laval ne partit pas pour le Tonkin. «Le projet lésait certains intérêts. Sous la pression du Portugal et de quelques Ordres religieux qui revendiquaient pour eux une sorte de monopole de l'évangélisation d'Extrême-Orient, la Propagande hésita et finalement classa la supplique ${ }^{28}$.» D'ailleurs, à Rome, on commençait à être ennuyé par les patronats d'exclusivité qu'exerçaient certains pays sur les territoires missionnaires, et la Propagande tentait précisément à cette époque d'arracher à tout contrôle son action en pays de mission ${ }^{29}$.

Les propositions du P. de Rhodes furent donc momentanément écartées. Ce ne fut que plus tard, en 1658, qu'elles furent réalisées. Mais alors les destinées de François de Laval avaient pris une autre direction.

Les désirs d'apostolat missionnaire de l'abbé de Montigny avaient donc échoué. "Il ne faut pas, disait M. de Bernières, se produire avant le temps. Ceux qui s'exposent à travailler pour le prochain avant d'être morts à eux-mêmes, font peu de fruit et risquent de se perdre ${ }^{30}$." Faisant mourir en lui ses ardents désirs de la gloire de Dieu, Laval se soumit à la Volonté divine et, dans la paix, attendit d'autres indications de la Providence.

Ces indications n'allaient pas tarder.

Il y avait longtemps que l'envoi d'un évêque dans les lointaines missions du Canada hantait les esprits apostoliques. Déjà, en 1631, le dynamique $\mathrm{M}^{\mathrm{gr}}$ François Ingoli, premier secrétaire de la Propagande, avait élaboré et soutenu avec fougue un projet pour l'érection d'un évêché en Nouvelle-France. Mais l'entreprise était, pour le moins, prématurée, même si elle dénotait l'intérêt que le Saint-Siège prenait pour les missions canadiennes ${ }^{31}$.

De nouvelles instances avaient été faites dans la suite par les Associés de Montréal en 1645, par l'Assemblée du clergé de France en 1646, par la Compagnie des Cent-Associés en 1650; mais toutes, pour des raisons dont l'exposé dépasserait les limites de ce travail, avaient échoué, laissant de côté les candidats proposés, tant Récollets, Jésuites, Sulpiciens que prêtres séculiers ${ }^{32}$.

avait une grande vénération. Faut-il penser qu'il alla, avec ses deux compagnons désignés pour l'épiscopat en Extrême-Orient, la consulter sur sa vocation de futur évêque missionnaire? $M$. le chanoine Eugène Lelièvre, dans une note qu'il nous a communiquée, le croit; mais son interprétation plutôt fantaisiste des documents sur lesquels il s'appuie, ne nous donne pas la certitude historique nécessaire pour que nous puissions le soutenir.

28 Coste, Monsieur Vincent, Paris, 1932, vol. III, p. 329.

29 Bernard de Vaulx, Histoire des missions françaises, Paris, 1951, pp. 134-138.

30 Latour, op. cit., pp. 6-7.

31 Conrad-M. Morin, o.f.m., Les tentatives du secrétaire François Ingoli pour l'érection d'un évêché au Canada, [0ttawa, 1945.].

32 [Michel-Etienne Faillon], Histoire de la colonie française en Canada, Villemarie, 1865-1866, vol. II, pp. 47-53, 271-275, 313-314; de Rochemonteix, op. cit., vol. II, pp. 190-202. 
En 1657, la Nouvelle-France était plus que jamais mûre pour accueillir son premier évêque.

La colonie se consolidait sur des bases plus fermes. Les explorateurs français, grands affamés d'espaces, avaient piqué au loin (trop loin peut-être, eu égard la faible densité de la population) les jalons du nouveau pays, et les colons faisaient reculer la forêt devant leur entêtement à se bâtir un domaine. Le régime seigneurial, par les échanges de bons services entre seigneurs et censitaires, non seulement activait l'immigration de nouveaux colons, mais fournissait des cadres à la société naissante et assurait l'avenir du pays. L'économie canadienne, encore branlante peut-être, aspirait déjà, par un système autarchique embryonnaire, à se suffire à elle-même.

D'autre part la mission canadienne, fécondée par le sang des martyrs, sentait pousser dans ses veines un regain de vitalité. Les missionnaires, désormais plus nombreux, assuraient aux colons les secours de la religion, et accompagnaient, quand ils ne les devançaient pas, les explorateurs, afin de fonder de nouveaux postes d'avant-garde chrétienne. Les communautés de femmes avaient ouvert des écoles pour l'éducation des enfants et créé des services hospitaliers. Bref, la jeune mission avait déjà élevé sa charpente d'Eglise. Il n'y manquait que la bénédiction d'un évêque pour consacrer et parfaire cette action colonisatrice et missionnaire.

Par ailleurs il y avait aussi bien des abus qui commençaient à se glisser dans le peuple. "Le vice commençait à s'y répandre," dit Latour ${ }^{33}$. Et l'on songe particulièrement à ce funeste commerce de l'eau-de-vie avec les Indiens, qui entraînait avec lui tant de désordres. Les missionnaires étaient alarmés et sentaient bien la nécessité urgente d'une vive réaction, que seule une autorité ecclésiastique bien définie pourrait exercer.

Or la mission canadienne n'avait précisément pas cette autorité ecclésiastique bien définie; on allait même en certains milieux jusqu'à se poser des questions sur la validité canonique des pouvoirs tenus par les missionnaires et des actes posés par eux.

Les premiers missionnaires venus en Nouvelle-France, les Récollets en 1615 , les Jésuites en 1625 et en 1632, avaient tout d'abord obtenu leurs pouvoirs ecclésiastiques du Saint-Siège, les premiers, par l'intermédiaire du Nonce de Paris ${ }^{34}$, et les seconds par celui de leur Général ${ }^{35}$. Or, voici que l'Archevêque de Rouen prétendit peu à peu étendre sa juridiction en terre canadienne, alléguant que la plupart des navires en partance pour le Canada faisaient voile de quelqu'une des villes de son diocèse et qu'il donnait aux prêtres qui voyageaient sur ces vaisseaux, des pouvoirs pour le temps de la traversée et pour le lieu de débarquement.

33 Latour, op. cit., p. 10.

34 Conrad-M. Morin, o.f.m., Le Saint-Siège et la juridiction des fondateurs de la mission du Canada, dans Antonianum, vol. XX (Roma, 1945), pp. 147-176. 35 de Rochemonteix, op. cit., vol. II, pp. 189-191. 
Les Jésuites, seuls missionnaires à partir de 1632 , ne s'en préoccupèrent pas d'abord; mais des lettres venues de la métropole, dans lesquelles on mettait en doute leurs pouvoirs, finirent par provoquer chez eux de sérieux scrupules sur la validité des mariages et autres actes de juridiction dans un pays où il n'y avait aucune autorité officiellement accréditée par Rome. Sachant que l'Archevêque de Rouen était en France de plus en plus reconnu comme l'Ordinaire de leurs missions, ils demandèrent et obtinrent, en 1649, des lettres patentes de grand vicaire, signées par l'Archevêque de Rouen, pour leur supérieur de Québec ${ }^{36}$; geste que d'ailleurs le Général de la Compagnie de Jésus ne voulut pas approuver. En 1657, un second vicaire général du même Archevêque de Rouen était nommé dans la personne de M. de Queylus, supérieur des Sulpiciens de Montréal.

La présence simultanée de deux vicaires généraux sans juridiction délimitée pouvait amener des conflits. En fait les conflits éclatèrent et quelques escarmouches s'en suivirent. Même si, en 1658, une trêve relative avait établi un moment de paix, on n'en désirait pas moins la nomination d'un évêque qui vînt clarifier la situation en faisant l'unité de juridiction sous sa houlette pastorale ${ }^{37}$.

Qui prendrait la direction de cette jeune Eglise et la gouvernerait avec assez de fermeté pour réprimer les abus et assez de tact pour régler les conflits?

Les Associés de Montréal, secondés par l'Assemblée du clergé de France, soutenaient la candidature de M. de Queylus ${ }^{38}$. Les Jésuites de leur côté proposaient à l'épiscopat de la Nouvelle-France leur ancien élève de La Flèche et de Clermont, l'abbé François de Laval. C'est ce dernier candidat que la Cour agréa pour le soumettre ensuite à la nomination du Saint-Siège.

Selon le P. de Rochemonteix, le P. Jean Bagot eut le premier la pensée de présenter comme premier évêque de Québec celui que, peu d'années auparavant, il avait suggéré comme vicaire apostolique au Tonkin. Le P. Annat, confesseur du roi, en parla au jeune Louis XIV, et le P. Paul Lejeune, procureur à Paris des Jésuites du Canada, fit la même proposition à la reine-mère Anne d'Autriche ${ }^{39}$.

La puissante Compagnie du Saint-Sacrement aurait-elle, à cette occasion, appuyé de son prestige à la Cour cette proposition qui était

36 Arch. générales de la Compagnie de Jésus, Gallia 109 IV, ff. 506-507.

37 Sur ces conflits de juridiction, cf. Faillon, op. cit., vol. II, pp. 278-282, 289 295, 297-302; de Rochemonteix, op. cit., vol. II, pp. 202-237; Conrad-M. Morin, o.f.m., Le Saint-Siège et l'établissement de l'Eglise du Canada, La mission apostolique (1615-1658), III ${ }^{\mathrm{e}}$ partie, chap. III (Ce chapitre de l'ouvrage du P. Morin n'a malheureusement pas encore été édité); Wilfrid-H. Paradis, Les archevêques de Rouen et l'établissement de la hiérarchie ecclésiastique au Canada au XVII siècle, (Nous regrettons que l'auteur n'ait publié qu'une partie de son travail: L'érection du diocèse de Québec et l'opposition de l'archevêque de Rouen, 1662-1674, dans Revue d'histoire de l'Amérique française, vol. IX (1955-1956), pp. 465-501).

38 Faillon, op. cit., vol. II, pp. 270-275.

39 de Rochemonteix, op. cit., vol. II, p. 278, note 1. 
faite de François de Laval comme évêque de Québec? Le mystère qui entoure encore bien des activités secrètes de la Compagnie et la pénurie de documents sur le point précis qui nous occupe, ne nous permettent pas de l'affirmer péremptoirement. Cependant cette intervention est très plausible, la Compagnie ayant si fortement secondé toutes les entreprises missionnaires de l'époque ${ }^{40}$, et peut-être expliquerait-elle cette lettre que $\mathrm{M}^{\mathrm{gr}}$ de Laval adressera de Québec en 1659 au secrétaire de la Compagnie, René de Voyer d'Argenson, et que celui-ci signale dans les annales de la société: "Le $28^{\circ}$ de décembre 1659 , écrit-il, la Compagnie reçut une lettre de $M$. l'évêque de Pétrée, qui l'assurait de son estime et que, bien qu'il fût séparé d'elle de plus de 1,200 lieues, il ne le serait jamais d'esprit. Je fus chargé, ajoute-t-il, de lui faire réponse au nom de la Compagnie et de le remercier des marques qu'il lui donnait de son affection la plus constante "(Annales, p. $110 \mathrm{~b}$ ) ${ }^{41}$.

Rappelons en passant que de cette note inscrite par d'Argenson dans ses Annales, et aussi du fait que François de Laval a été pendant quatre ans un hôte assidu de l'Ermitage de Caen, considéré à bon droit comme une succursale de la Compagnie du Saint-Sacrement ${ }^{42}$, on a inféré que le premier évêque de Québec a lui-même appartenu à la Compagnie. Cependant l'argument ne nous semble pas probant. Au reste, Raoul Allier, qui a tenté de dresser une liste des membres de cette société, n'a inscrit qu'avec doute le nom de François de Laval ${ }^{43}$, et Madame Louise C. Rosett, de Washington, qui effectue actuellement des études très poussées sur la mystérieuse Compagnie du Saint-Sacrement, ne croit pas que $\mathrm{M}^{\mathrm{gr}}$ de Laval en ait jamais fait partie. On donnait d'ailleurs "l'entrée libre » à certains évêques amis ou protégés de la Compagnie, mais non inscrits parmi ses membres ${ }^{44}$. Peut-être $M^{g r}$ de Laval a-t-il été de ceux-là.

Au reste François de Laval n'était pas un inconnu à la Cour. Son nom se référait à deux grandes maisons figurant en première place dans l'armorial de la haute noblesse de France. D'autre part l'on avait été à même, tout dernièrement encore, de juger, dans certains milieux, de son intelligence et de son jugement.

En effet l'évêque de Bayeux, $M^{\text {gr }}$ François Servien, dans le diocèse duquel se trouvait l'Ermitage de $M$. de Bernières, l'avait récemment envoyé à Paris pour défendre les intérêts des religieuses de l'hôpital de Caen contre les poursuites des administrateurs de ce même hôpital. François de Laval plaida si bien qu'il obtint gain de cause sur tous les points ${ }^{45}$. Son biographe, Bertrand de Latour, assure que "son voyage

40 Georges Goyau, Les prêtres des missions étrangères, Paris, 1932, pp. 43-64.

41 Cité par Raoul Allier, La Compagnie du Très Saint-Sacrement. La cabale des dévots, Paris, 1902, pp. 148-149.

42 Maurice Souriau, op. cit., pp. 55-56.

43 Raoul Allier, op. cit., pp. 34-37.

44 L. Carey Rosett, A la recherche de la Compagnie du Saint-Sacrement à Montauban, dans Revue d'histoire de l'Eglise de France, vol. XL (juill.déc. 1954), p. 220, note 52 .

45 Mémoire touchant feu M. François de Laval, 1708, Arch. du Séminaire de Saint-Sulpice de Paris, Documents pour servir..., liasse de papiers Laval, fol. 3 . 
le fit connaître à la Cour. Son nom lui ouvrit les avenues et sa vertu lui gagna tous les cœurs ${ }^{46}$ ".

Ce fut le jeune roi Louis XIV (il avait alors dix-huit ans) qui, encouragé et soutenu par la reine-mère Anne d'Autriche, prit l'initiative de doter l'Eglise canadienne de son premier évêque.

Le 26 janvier 1657, il écrivait une lettre personnelle au pape Alexandre VII pour le prier d'ériger un évêché en Nouvelle-France et d'y nommer comme premier titulaire « le Père François de Laval, dont les vertus, disait-il, l'ont rendu si recommandable ${ }^{47}$ \%.

En même temps, le souverain confiait à son agent en Cour de Rome, le sieur Gueffier, le soin de presser l'affaire auprès du Saint-Siège en vue d'obtenir au plus tôt la faveur implorée ${ }^{48}$.

Cependant malgré les sollicitudes très empressées de l'agent royal et malgré l'intervention des Pères Jésuites de la maison générale ${ }^{49}$, les difficultés et les complications surgirent si nombreuses que les tractations traînèrent en longueur pendant plus d'un an.

La maladie du cardinal Bichi, que Louis XIV avait prié de présenter au Pape sa lettre de requête, et qui était retenu à la chambre souffrant de la goutte ${ }^{50}$;

la peste qui sévit à Rome au printemps de 1657 et qui empêcha les réunions régulières des Congrégations romaines ${ }^{51}$;

l'erreur commise par le roi en attribuant le nom de Père à François de Laval; ce qui exigea l'échange de plusieurs lettres entre Rome et Paris pour assurer que le candidat proposé à l'évêché de Québec n'appartenait à aucune communauté religieuse ${ }^{52}$;

le projet utopique entretenu par la Propagande de fonder au Canada cinq provinces ecclésiastiques dans les cinq principales régions habitées par les Indiens ${ }^{53}$;

C'est en partie à travers le dossier des lettres envoyées par de Gueffier au comte de Brienne, alors ministre d'Etat de Louis XIV et chargé des affaires étrangères, que l'on peut suivre le développement de ces négociations. Ces lettres sont conservées au British Museum de Londres, Fonds Harley, 4541 $\mathrm{A}$; c'est à ce fonds que renvoient toutes nos citations de cette correspondance. On trouvera également la transcription de ces lettres dans de Rochemonteix, op. cit., vol. II, pp. 470-501.

49 de Rochemonteix, op. cit., vol. II, p. 282, note 1.

50 Gueffier à Brienne, 22 février 1657, loc. cit., ff. 43-45; 5 mars 1657, loc. cit., ff. 47-49.

51 Gueffier à Brienne, 19 mars 1657, loc. cit., fol. 58.

52 Gueffier à Brienne, 19 mars 1657, loc. cit., ff. 57-58; Brienne à Gueffier, 27 avril 1657, copie aux Arch. de la Propagande, Scritture riferite nelle cong. generali, vol. 317, fol. 103; Gueffier à Brienne, 15 mai 1657, loc. cit., fol. 82 ; 29 mai 1657 , loc. cit., fol. 88.

53 Faillon, op. cit., vol. II, pp. 319-320. 
la demande, la rédaction et l'expédition par le nonce de Paris Celio Piccolomini de nouvelles Informations canoniques sur la vie et les mœurs de l'abbé François de Laval ${ }^{54}$;

l'intervention des Jésuites pour obtenir une nomination de vicaire apostolique plutôt que celle d'évêque résidentiel, la mission canadienne étant jugée encore trop jeune pour être élevée au rang d'évêché ${ }^{55}$;

la question de la pension annuelle de 1,000 Livres tournois que le roi promettait au nouvel évêque et dont le Pape voulait s'assurer ${ }^{\tilde{5} 6}$;

enfin les échanges de mémoires pour une rédaction équitable des bulles, qui satisfassent et les désirs du Souverain Pontife et les exigences de Sa Majesté très chrétienne ${ }^{57}$;

bref les complications semblaient accumuler à plaisir les contretemps pour exaspérer la patience du chargé d'affaires royal et retarder l'issue des négociations.

Si bien que ce ne fut que le llavril 1658 que la Sacrée Congrégation de la Propagande émit enfin le décret nommant François de Laval évêque de Pétrée in partibus infidelium et vicaire apostolique en la Nouvelle-France ${ }^{58}$; deux jours plus tard, soit le 13 avril, Alexandre VII donnait son placet ${ }^{59}$, et le 6 mai suivant le nouvel élu était enfin proclamé en consistoire ${ }^{60}$.

Que faisait pendant ce temps François de Laval ?

Tout simplement il attendait les dispositions du Seigneur sur sa vie; " martyr de la longanimité ", dira un jour avec humour un de ses amis dans une circonstance analogue ${ }^{61}$.

Il passe la plus grande partie de son temps à l'Ermitage de Caen, exact aux exercices spirituels des fervents ermites. Fidèle à une maxime très chère à $M$. de Bernières: " $O n$ ne trouve la vie que dans la mort ${ }^{62}$," il s'applique à faire mourir en lui toute ambition terrestre pour mieux se disposer à sa vie de futur missionnaire.

54 Informations canoniques, 17 juillet 1657 , copie authentiquée par $M^{\mathrm{gr}}$ de Laval, Arch. du Séminaire de Québec, Evêques de Québec, $\mathrm{n}^{\circ} 183$.

55 Gueffier à Brienne, 17 décembre 1657, loc. cit., ff. 224-225; Faillon, op. cit., vol. II, pp. 320-321.

56 Gueffier à Brienne, 7 janvier 1658, loc. cit., fol. 243; 21 janvier 1658, loc. cit., fol. 274; copie du mémoire sur le revenu promis à $M^{\mathrm{gr}}$ de Laval, ibid., fol. 296; rapport et rescrit de la Propagande, Arch. de la Propagande, Acta, vol. 27, fol. 94; l'original de la constitution de cette rente, signé par Anne d'Autriche, se trouve aux Archives du Séminaire de Québec, Séminaire II, $n^{\circ} 3$.

57 Gueffier à Brienne, 12 juin 1657, loc. cit., fol. 97; 19 juin 1657, loc. cit., fol. 99.

58 Cité par de Rochemonteix, op. cit., vol. II, pp. 498-499.

59 de Rochemonteix, op. cit., vol. II, p. 499.

60 Gueffier à Brienne, 6 mai 1658, loc. cit., fol. 325.

61 Gazil à Pallu, $1^{\text {er }}$ mars 1675, Arch. du Séminaire des missions étrangères de Paris, vol. V, p. 551 .

62 Latour, op. cit., p. 7. 
A une époque où l'ambition suggérait à tant de clercs des démarches, et parfois de basses intrigues, pour obtenir un bénéfice ecclésiastique ou se voir décerner une mitre épiscopale, l'abbé de Montigny ne pose aucun acte susceptible d'accélérer les négociations en cours pour sa nomination d'évêque.

A Rome, le sieur Gueffer, agent du roi, qui s'agite et s'affaire pour mener à bonne fin cette entreprise, ne comprend rien à cette attitude peut-être nouvelle pour lui. Plusieurs fois il exprime sa surprise à son correspondant de Paris, le comte de Brienne ${ }^{63}$, et au moment où les pourparlers vont aboutir, il s'étonne, comme il le dit lui-même, de ce que « le sieur de Montigny... ne lui ait jamais écrit un mot en ce qui regarde en cela son service ${ }^{64}$ ».

Pourtant l'offre qui lui était faite de devenir missionnaire devait combler pour François de Laval des désirs déjà anciens. Sa situation familiale et le bon renom de ses vertus auraient bien pu, il est vrai, le conduire sur un trône épiscopal de France; mais, comme l'écrivait de lui Louis XIV au pape Alexandre VII, "par des mouvements secrets, il se sentait porté d'aller plutôt dans un pays sauvage qu'en un civilisé et abondant en toutes choses nécessaires à la vie; ce qui, d'ajouter le roi, ne se trouve que difficilement en Nouvelle-France ${ }^{65}$ ".

La Nouvelle-France, elle avait jadis hanté les rêves du jeune étudiant au collège de La Flèche. Voici qu'elle reparaissait à l'horizon de sa vie.

Peut-être même lui offrait-elle de combler ce désir du martyre qui se développait dans son âme généreuse; de ce désir du martyre, il donnera une expression non équivoque dans une lettre qu'il adressera au Général de la Compagnie de Jésus au lendemain de son arrivée à Québec: "Je m'estime trop heureux, avouera-t-il alors au souvenir de ses maitres immolés pour le Christ en pays huron, je m'estime trop heureux de pouvoir espérer le même sort et de devenir participant de leur couronne, de subir la même mort, non il est vrai en vertu de mes mérites, mais par la libéralité et une faveur gratuite du Dieu compatissant ${ }^{66}$."

La Nouvelle-France, elle l'appelait maintenant d'une voix plus pressante. Mais elle lui proposait en même temps un honneur qui répugnait à son humilité toujours en progrès à l'école de $M$. de Bernières, celui de l'épiscopat. "Je n'ai jamais désiré d'épiscopat, écrira-t-il en 1672 aux Cardinaux de la Propagande, et c'est conscient de ma faiblesse que je l'ai accepté malgré moi ${ }^{67}$." Les contemporains de sa vie appor-

63 Gueffier à Brienne, 15 mai 1657, loc cit., fol. 82; 29 mai 1657, loc. cit., fol. 88 ; 30 juillet 1657, loc. cit., fol. 137; 6 mai 1658, loc. cit., fol. 323 .

64 Gueffier à Brienne, 6 mai 1658, loc. cit., fol. 323.

65 Louis XIV à Alexandre VII, 26 janvier 1658, Arch. Vat., Lettere di principi, vol. 81, fol. 4.

$66 \mathrm{M}^{\mathrm{gr}}$ de Laval au P. Goswin Nickel, août 1659, Arch. générales de la Compagnie de Jésus, Gallia 109 II, fol. 436.

67 Arch. de la Propagande, Scritture riferite nelle cong. gen., vol. 433, fol. 691. 
teront semblable témoignage recueilli sans aucun doute sur la bouche $\mathrm{du}$ prélat. Il avait même fallu l'intervention de ses amis et tout l'ascendant qu'exerçait sur lui $M$. de Bernières pour le convaincre d'accepter un honneur et une charge dont il se sentait indigne et incapable ${ }^{68}$.

Finalement vers le début du mois d'août 1658, François de Montmorency-Laval, probablement alors à Paris ${ }^{69}$, reçut les bulles pontificales qui le nommaient évêque de Pétrée in partibus infidelium et lui confiaient la charge de vicaire apostolique en Canada ${ }^{70}$. Elles étaient datées du 3 juin précédent, mais n'avaient pu être expédiées de Rome avant le 7 juillet ${ }^{71}$.

Dans ces bulles concédées par le Pape Alexandre VII, l'évêque-élu vit un ordre définitif de la Providence; dès lors il n'hésita plus.

Il se retira de nouveau à l'Ermitage de Caen ${ }^{72}$, où, tout en continuant de disposer son âme à sa consécration épiscopale, il vit en même temps à régler les détails de la cérémonie prochaine.

Il avait choisi le 4 octobre, fête de saint François d'Assise, pour le jour de son sacre, et c'est son ami, $M^{\text {gr }}$ François Servien, évêque de Bayeux, qui devait lui donner la plénitude du sacerdoce, assisté de $\mathbf{M}^{\mathrm{gr}}$ l'évêque d'Ardue ${ }^{73}$ et de $M^{\mathrm{gr}}$ l'évêque d'Evreux ${ }^{74}$.

Or il arriva qu'au matin même du jour fixé pour la cérémonie (du moins c'est ce que prétend Bertrand de Latour) ${ }^{75}$, l'évêque d'Ardue reçut de $\mathrm{M}^{\mathrm{gr}}$ François de Harlay de Champollion, archevêque de Rouen, dont il était le suffragant, la défense expresse d'y participer. De son côté, l'évêque d'Evreux avait eu communication d'une défense semblable de la part de l'Assemblée des évêques de France ${ }^{76}$.

Que s'était-il donc passé ?

Depuis vingt-cinq ans déjà, comme nous l'avons signalé plus haut, l'Archevêque de Rouen avait été amené, par une sorte d'usurpation de pouvoir et par une succession de faits, à considérer le Canada comme un prolongement de son diocèse. On comprend dès lors son étonnement

68 Avis particuliers de M. de Bernières pour M. de Laval, dans Latour, op. cit., p. 28; de la Colombière, Eloge funèbre de $\mathbf{M}^{\mathrm{gr}}$ de Laval, Archives du Séminaire de Nicolet; Mémoire touchant feu M. François de Lavai, 1708, Arch. du Séminaire de St-Sulpice de Paris, Documents pour servir..., vol. I, liasse

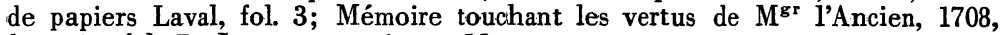
loc. cit., fol. 7; Latour, op. cit., p. 11.

69 Latour, op. cit., p. 12.

70 Bulles de nomination de $\mathrm{M}^{\mathrm{gr}}$ de Laval, Arch. de l'Archevêché de Québec.

71 Gueffier à Brienne, loc. cit., fol. 348 .

72 Latour, loc. cit.

73 Latour, loc. cit.

74 Auguste Gosselin, Vie de $M^{g r}$ de Laval, Québec, 1890, vol. II, p. 123.

75 Latour, loc. cit.

76 Sur cette opposition apportée au sacre de $M^{\mathrm{gr}}$ de Laval, cf. Faillon, op. cit., vol. II, pp. $323-326$. 
lorsqu'il apprit, sans en avoir été prévenu (il semble que toutes les négociations entre Rome et Paris aient été menées dans le plus grand secret) qu'un administrateur apostolique était envoyé par le Pape dans un territoire qu'il soutenait être soumis à son autorité épiscopale.

Soit qu'il conçût certaines préventions contre le titre d'évêque in partibus et contre les droits de vicaire apostolique, soit qu'il mît en doute la légalité des bulles accordées à $M^{\mathrm{gr}}$ de Laval, soit qu'il se fût entêté à soutenir contre Rome ses droits prétendus de juridiction sur la Nouvelle-France, il obtint d'une part de l'Assemblée des évêques de France un ordre prohibant à tout évêque du royaume de consacrer le nouveau prélat, et d'autre part du Parlement de Rouen une défense intimée à l'évêque-élu d'exercer aucun pouvoir de vicaire apostolique au Canada.

Surpris, les trois évêques consécrateurs durent se retirer, et la cérémonie n'eut pas lieu.

$M^{\mathrm{gr}}$ de Laval partit alors pour Paris et remit toute l'affaire entre les mains du Nonce apostolique, qui en déféra au Saint-Siège.

A Rome, les cardinaux de la Propagande sursautèrent. Ils allèrent même jusqu'à proposer au Nonce de Paris de faire lui-même la consécration épiscopale du futur vicaire apostolique, assisté, si aucun évêque français ne voulait accepter son invitation, de deux abbés, de deux chanoines ou de deux simples prêtres ${ }^{77}$. D'ailleurs le Pape avait déjà fait avertir, par voie diplomatique, l'Archevêque de Rouen de vouloir bien se désister de toute prétention juridique sur le Canada ${ }^{78}$.

Anne d'Autriche, à qui la nomination de $\mathrm{M}^{\mathrm{gr}}$ de Laval tenait à cœur, intervint personnellement auprès du Nonce et le pria de donner, sans délai et dans le plus grand secret, la consécration épiscopale au nouvel évêque ${ }^{79}$.

Le Nonce choisit pour la cérémonie l'abbaye bénédictine de SaintGermain-des-Prés, située alors dans la banlieue parisienne et exempte de la juridiction épiscopale de l'Archevêque de Paris.

Le dimanche, 8 décembre 1658, en la fête de l'Immaculée Conception, envers laquelle il conserva toujours une particulière dévotion, François de Montmorency-Laval, évêque-élu de Pétrée in partibus infidelium et vicaire apostolique nommé de la Nouvelle-France, recevait secrètement la consécration épiscopale des mains de $\mathrm{M}^{\mathrm{gr}}$ Celio Piccolomini, Nonce apostolique à Paris ${ }^{80}$. Le rite sacré se déroula, non dans l'église de l'abbaye ${ }^{81}$, mais dans la grande chapelle gothique de la

77 Faillon, op. cit., vol. II, p. 327.

78 Gueffier à Brienne, 10 décembre 1658, loc. cit., ff. 420-421.

79 ...havendo la regina fattomi dire di farla (la consacrazione), , Le Nonce au Secrétaire d'Etat, 20 décembre 1658, Arch. Vat., Nunziatura di Francia, vol. 113, fol. 479.

80 Mémoire touchant feu M. de Laval, 1708, loc. cit., fol. 3; Latour, op. cit., 12-15.

81 Un bas-relief en pierre polychrome, œuvre du sculpteur Henri Charlier, rappelant le sacre de $\mathrm{M}^{\mathrm{gr}}$ de Laval, a été érigé dans l'église de SaintGermain-des-Prés le 11 août 1923, à l'occasion des fêtes qui ont marqué le troisième centenaire de la naissance du premier évêque de Québec. 
Vierge ${ }^{82}$ construite en 1245 dans l'enceinte du monastère par Pierre de Montreuil, le même architecte qui bâtit la Sainte Chapelle dans l'île de la Cité.

Les deux évêques qui, vraisemblablement sur demande de la reine, avaient accepté d'assister le pontife, étaient $\mathrm{M}^{\mathrm{gr}}$ Hardoin de Préfixe (et non $\mathbf{M}^{\mathrm{gr}}$ Louis Abelly, comme on l'a dit communément) ${ }^{83}$ et $\mathrm{M}^{\mathrm{gr}}$ André du Saussay, évêque de Toul ${ }^{84}$.

Quels furent les sentiments intimes qui envahirent l'âme du jeune évêque de trente-six ans, lorsqu'il reçut la plénitude du sacerdoce, nous ne saurions le dire. $M^{\mathrm{gr}}$ de Laval a toujours été très réservé dans ses confidences de vie spirituelle. Du moins l'histoire n'a conservé que peu de documents capables de nous livrer directement cet aspect de sa personnalité, et ce n'est qu'à travers des réflexions passagères semées au cours de ses écrits qui nous sont parvenus, et par ses actes, qu'on peut suivre, et encore difficilement, le cheminement de son âme vers les sommets de la perfection chrétienne. De ses impressions de sacre, nous ne savons rien.

Un peu plus expansif de ses sentiments fut l'un de ceux qui avec le Nonce lui imposèrent les mains pour l'épiscopat et qui, au dire du P. Jérôme Lallemant, répétait souvent avoir eu moins l'impression de sacrer un évêque que de consacrer un martyr ${ }^{85}$.

A l'Ermitage de Caen, M. de Bernières exulta de joie quand il apprit le sacre de $\mathrm{M}^{\mathrm{gr}}$ de Laval; et l'on sent dans sa lettre du 12 décembre 1658 au nouvel évêque tout le soin assidu que le maître avait mis pour entretenir son illustre disciple dans un grand esprit d'abandon confiant à la Providence, au moment où s'était décidé son avenir. "Je ne puis vous exprimer, écrivait le directeur de l'Ermitage, la joie que nous avons

82 Dom Jacques Bouillart, Histoire de l'abbaye de Saint-Germain-des-Prez, Paris, 1724, liv. V, p. 256 (cité par Auguste Gosselin, Henri de Bernières, Evreux, 1897, p. 9, note 1).

83 C'est par erreur que Latour (op. cit., p. 12), suivant peut-être le Mémoire touchant feu M. de Laval (loc. cit., fol. 3), nomme . M. Abelli, évêque de Rodez» comme co-consécrateur de $M^{\mathrm{gr}}$ de Laval. En fait Louis Abelly n'était pas encore évêque de Rodez; il occupait à cette époque le poste de directeur spirituel de l'Hôpital Général de Paris. Par ailleurs le Nonce affirme expressément au Secrétaire d'Etat qu'il a fait la cérémonie * con l'assistenza di Mons. di Rodez, precettore del re (Arch. Vat., Nunziatura di Francia, vol. 113, fol. 470). Or, en 1658, l'évêque de Rodez était, depuis le 6 janvier précédent, Hardouin de Préfixe, qui avait été précédemment précepteur du jeune Louis XIV et qui sans doute gardait encore ce titre. Nommé archevêque de Paris le 30 juillet 1662 et confirmé à ce siège le 24 mars 1664, il mourut le $1^{\text {er }}$ janvier 1671 (Gambs, Series episcoporum, Ratisbonne, 1873, pp. 597, 613). Sur cette question, voir aussi G. de LacourtGayet, Le sacre du premier évêque de Québec, dans Revue des jeunes, vol. XXXVII (1923), pp. 508-519.

84 André du Saussay, nommé évêque de Toul en 1649, consacré le 16 juillet 1656 et mort le 9 septembre 1675 (Gambs, op. cit., p. 636).

85 "Ex is unus qui ipsum in episcopum inunxit dicere solitus est se eum ipsum ungere putasse non tam episcopum quam martyrem consecrare; forte non falsus vates ", Le P. Jérôme Lallemant au Général de la Compagnie de Jésus, Archives générales de la même Compagnie, Gall. 109 I, fol. 310. 
tous reçue d'apprendre par vos chères lettres votre sacre, qui a été fait sans doute par une providence toute particulière de Dieu. Vous pouvez tout, Monseigneur, en Celui qui vous conforte. Ne quittez jamais, permettez-moi de vous parler de la sorte, cette manière d'agir en esprit d'anéantissement. Dans le grand emploi que Notre-Seigneur met sur vos épaules, et dans toute la conduite de votre vie, ne vous comportez jamais autrement... Votre âme trouvera des trésors immenses dans cette sainte pratique de l'anéantissement... Nous aurions grande consolation, ajoutait-il, de pouvoir encore vous voir une fois avant de quitter la France, afin de parler à cœur ouvert du divin état d'anéantissement ${ }^{86}$.»

Cependant cette nouvelle de la consécration épiscopale de $\mathrm{M}^{\mathrm{gr}}$ de Laval, faite secrètement par le Nonce, ne produisit pas, on peut l'imaginer, partout le même effet. En certains milieux, hostiles à la promotion du nouvel évêque, elle fut reçue comme un coup de foudre, prélude d'une nouvelle tempête.

L'Archevêque de Paris fut offusqué de ce que le Nonce avait présidé à la cérémonie sans l'en avoir avisé, dans une église exempte, il est vrai, de son autorité épiscopale, mais qui était située dans les limites de son diocèse ${ }^{87}$.

L'Archevêque de Rouen, qui n'avait pu empêcher par ses intrigues le sacre du nouveau prélat, résolut de tout mettre en œuvre pour arrêter son départ vers la Nouvelle-France. Il protesta auprès de l'agent général de l'Assemblée du clergé, l'abbé Thoreau, et auprès du cardinal de Mazarin, sympathique à sa cause ${ }^{88}$, allant jusqu'à exiger que le vicaire apostolique, s'il devait réellement passer en Canada, prît de lui des pouvoirs de vicaire général avant d'exercer son ministère ${ }^{89}$.

Le Parlement de Paris, sur requête du procureur général, fit assigner à $\mathrm{M}^{\mathrm{gr}}$ de Laval une sommation de comparaître devant lui pour " rapporter et lui communiquer les prétendus brefs et bulles obtenus, exécutés sans la permission du roi ", avec défense "de s'immiscer à l'exécution d'icelles sans les avoir préalablement présentées au dit seigneur roi et obtenu sur ce lettres patentes en la manière accoutumée ${ }^{90}$ ».

Le Parlement de Rouen signifia lui aussi une défense du même genre au vicaire apostolique ${ }^{91}$.

Dans ce concert de protestations, on ignorait, ou du moins on feignait d'ignorer, que c'était sur requête de Louis XIV et d'Anne

86 Cité par Auguste Gosselin, Henri de Bernières, Evreux, 1896, p. 9.

87 Latour, op. cit., pp. 13-16.

88 L'abbé Thoreau au cardinal de Mazarin, 10 décembre 1658, Arch. du ministère des affaires étrangères de Paris, Correspondance politique, Rome, vol. 133, fol. 599.

89 L’Archevêque de Rouen à Mazarin, 10 décembre 1658, loc. cit., fol. 596; vol. 137, ff. 105-106.

90 Extrait des registres du Parlement de Paris, 16 décembre 1658, loc. cit., vol. 133, fol. 608; Latour, op. cit., pp. 16-19.

91 Latour, op. cit., pp. 14-15. 
d'Autriche que le Saint-Siège avait nommé François de MontmorencyLaval, et que le Nonce n'avait sacré le prélat que sur demande expresse de la reine.

Aussi le souverain et "sa très honorée Dame et mère » parurent-ils un peu dans l'embarras.

En vue de satisfaire le Nonce, qui demandait la cassation de l'arrêt émis par le Parlement de Paris ${ }^{92}$ et voulait éviter au nouvel évêque l'humiliation d'une comparution devant les tribunaux, on chercha des compromis ${ }^{93}$; mais pour couper court à toute discussion, Louis XIV agréa purement et simplement, mais publiquement cette fois, la nomination de $\mathrm{M}^{\mathrm{gr}}$ de Laval ${ }^{94}$.

Le roi donna alors au vicaire apostolique des lettres patentes, datées du 27 mars 1659, qui l'autorisaient à exercer sa juridiction épiscopale dans les limites de la Nouvelle-France; ces lettres, le jeune évêque devait les présenter au gouverneur du Canada dès son arrivée dans le pays. Le roi y affirme expressément: "Nous avons de notre autorité royale déclaré et nous déclarons par les présentes signées de notre main, que nous voulons et qu'il nous plaît que le sieur de Laval de Montigny, évêque de Pétrée, soit reconnu par tous nos sujets dans les dites provinces pour faire les fonctions épiscopales sans préjudice des droits de la juridiction ordinaire ${ }^{95}$.»

"Sans préjudice des droits de la juridiction ordinaire...» La formule était équivoque et laissait encore à l'Archevêque de Rouen le loisir de conserver ses prétentions de juridiction ecclésiastique sur la Nouvelle-France. D'autant plus que Louis XIV ajoutait: «Et cela, en attendant l'érection d'un évêché dont le titulaire sera suffragant de l'Archevêque de Rouen." Ce que en fait Rome ne voulait pas accepter.

Le Nonce Piccolomini avait pourtant fait remarquer à Mazarin, qui avait rédigé le texte des lettres patentes ${ }^{96}$, que c'était, somme toute, reconnaître en même temps deux autorités. Le ministre n'en tint aucun compte, et le roi signa le document.

Serait-ce pour réparer cette erreur de son fils, qu'Anne d'Autriche écrira le 31 mars 1659 une lettre personnelle au gouverneur d'Argenson, dans laquelle elle lui recommande " de faire reconnaître le sieur Evêque de Pétrée en qualité de vicaire apostolique dans tout le Canada..., à tenir la main qu'il soit obéi dans toutes les fonctions épiscopales... et faire repasser en France tous ceux qui voudront s'opposer à son établissement et ne pas se soumettre à sa juridiction " ${ }^{97}$ ".

92 Faillon, op. cit., p. 329.

93 L'abbé Thoreau au cardinal de Mazarin, 20 décembre 1658. Arch. du ministère des affaires étrangères de Paris, Correspondance politique, Rome, vol. 133, ff. 612-613.

94 Faillon, op. cit., p. 329.

9ã Copie conservée aux Arch. du Séminaire de Québec, Chapitre, $\mathrm{n}^{\circ} 263$

96 Un texte annoté de ce projet est conservé à la Bibl. Nat. de Paris, Fonds français, vol. 20784 , fol. 595 .

97 Arch. de l'archevêché de Québec, Registre $A, n^{\circ} 184$. 
Faut-il voir aussi l'influence de la reine-mère dans cette lettre que Louis XIV adressait également à M. d'Argenson, le 14 mai suivant, pour lui ordonner de "maintenir le sieur Evêque en la pleine fonction de sa charge, soit qu'on le considère honoré du caractère épiscopal, soit du vicariat apostolique, dont, écrit le roi, j'ai recherché Sa Sainteté ${ }^{98}$ ».

Quoi qu'il en soit, $M^{\text {gr }}$ de Laval entrera en Nouvelle-France avec des pouvoirs de juridiction apostolique rendus imprécis par l'attitude de tergiversation adoptée par la Cour, même si dans la pensée de l'évêque ils étaient clairement appuyés par l'autorité romaine.

Aussi dès son arrivée en terre canadienne, le vicaire apostolique aurat-il à dissiper des doutes surgis dans les esprits les mieux intentionnés ${ }^{99}$ et devra-t-il lutter pour se faire accepter de tous. Ce ne sera en définitive qu'en 1674, lorsque le Saint-Siège érigera le diocèse de Québec en toute indépendance de celui de Rouen, que son autorité épiscopale sera indubitablement reconnue et que l'archevêque de la capitale normande fera son deuil de ses vieilles prétentions sur l'Eglise du Canada ${ }^{100}$.

Pendant que se nouaient toutes les intrigues qui tendaient à entraver l'exécution des volontés du Saint-Siège à son sujet, $\mathrm{M}^{\mathrm{gr}}$ de Laval, une fois consacré, conservera toujours le calme et la sérénité que lui inspiraient sa confiance et son abandon au bon vouloir divin. "Il nous faut laisser conduire à la Providence de Dieu ", écrira-t-il un jour ${ }^{101}$. départ.

Mais il n'en travaille pas moins activement à préparer son prochain

Peu de temps après son sacre, nous le retrouvons à Caen pour la dernière fois auprès de $M$. de Bernières ${ }^{102}$. Dans des avis particuliers, qui prennent l'accent d'un testament spirituel, le directeur de l'Ermitage donna alors à son disciple des conseils de vie intérieure ${ }^{103}$, que ce dernier gardera comme un héritage sacré. Ils ne devaient plus se revoir. Quelques mois plus tard, le 17 mai 1659, M. de Bernières mourait à Caen ${ }^{104}$, au moment même où Laval inaugurait à Percé son ministère pastoral.

En revenant à Paris, $\mathrm{M}^{\mathrm{gr}}$ de Laval passa-t-il par Montigny-surAvre donner à sa mère sa bénédiction et lui faire ses adieux? L'abbé Auguste Gosselin le suppose; mais la discrétion habituelle de François de Laval n'a pas levé le voile sur ce détail de sa vie intime. Nous savons seulement que la mère mourut la même année 1659, peu de temps après le départ de son fils ${ }^{105}$.

\footnotetext{
98 Copie contemporaine, Arch. du Sémin. de Québec, Lettres $\mathrm{N}, \mathrm{n}^{\circ} 2$.

99 Annales de l'Hôtel-Dieu de Québec, édition Dom Jamet, Montréal, 1939, p. 105.

100 Wilfrid-H. Paradis, L'érection du diocèse de Québec et l'opposition de l'archevêque de Rouen, 1662-1674, dans Revue d'histoire de l'Amérique française, vol. IX (1955-1956), pp. 465-501.

101 Apostille à une lettre de M. Henri-Jean Tremblay aux prêtres du Séminaire de Québec, 10 mai 1695, Arch. du Séminaire de Québec, Lettres N, $\mathbf{n}^{\circ} 101$.

102 Auguste Gosselin, Vie de $M^{g r}$ de Laval, Québec, 1890, p. 137.

103 Latour, op. cit., pp. 28-29.

104 Maurice Souriau, op. cit., p. 120.

105 Auguste Gosselin, op. cit., vol. I, pp. 136-137.
} 
Après avoir prêté, avec l'autorisation du Saint-Siège, son serment de fidélité entre les mains du roi ${ }^{106}$, il quitta Paris pour Larochelle au début d'avril.

Le 13 avril 1659, jour de Pâques, le vicaire apostolique s'embarquait pour le Canada. Il était accompagné de quatre prêtres: M. Jean Torcapel, M. Philippe Pellerin, M. Charles de Lauzon-Charny, ancien administrateur du Canada et entré depuis dans les ordres, et le P. Jérôme Lallemant, recteur du collège de La Flèche et jadis missionnaire en Nouvelle-France. A ce petit troupeau s'était joint au tout dernier moment le propre neveu de M. de Bernières, Henri de Bernières, simple tonsuré, qui fut dans la suite premier curé de Québec et premier supérieur du Séminaire de Québec ${ }^{107}$.

A mesure que s'éloignaient dans le lointain bleuâtre les côtes de son pays, nous aimons à nous représenter le jeune évêque de trente-six ans debout à la proue du navire, face au grand vent qui lui apportait déjà le souffle de sa nouvelle patrie; son cœur d'apôtre entrevoyait, dans l'horizon sans fin, sa jeune Eglise, où il allait consolider par son labeur apostolique et peut-être, pensait-il, par son sang, la croix plantée par les pionniers de la Foi, et réaliser les rêves d'enfant qui jadis l'avaient si souvent transporté en esprit dans les forêts du Nouveau Monde.

Georges-Edouard DemERs, ptre.

106 Latour, op. cit., p. 18.

$107 \mathrm{M}^{\mathrm{gr}}$ de Laval à Alexandre VII, 31 juillet 1659, Arch. Vat., Vescovi, vol. 44, fol. 145; P. Jérôme Lallemant au Général des Jésuites, 16 septembre 1659, Arch, générales de la Compagnie de Jésus, Gallia 109 A, fol. 309; Latour, op. cit., pp. 21-22. 